\title{
Intra-operative MRI facilitates tumour resection during trans-sphenoidal surgery for pituitary adenomas
}

\author{
Jon Ramm-Pettersen • Jon Berg-Johnsen • \\ Per Kristian Hol • Sumit Roy • Jens Bollerslev • \\ Thomas Schreiner $\cdot$ Eirik Helseth
}

Received: 15 November 2010 / Accepted: 23 March 2011 /Published online: 27 April 2011

(C) The Author(s) 2011. This article is published with open access at Springerlink.com

\begin{abstract}
Background During trans-sphenoidal microsurgical resection of pituitary adenomas, the extent of resection may be difficult to assess, especially when extensive suprasellar and parasellar growth has occurred. In this prospective study, we investigated whether intra-operative magnetic resonance imaging (iMRI) can facilitate tumour resection. Methods Twenty patients with macroadenomas, (16 nonfunctioning, three growth-hormone secreting and one pharmaco-resistant prolactinoma) were selected for surgery in the iMRI. The mean tumour diameter was $27 \mathrm{~mm}$ (range 11-41). The mean parasellar grade, according to the Knosp classification, was 2.3. Pre-operative coronal and sagittal T1-weighted and T2-weighted images were obtained. The trans-sphenoidal tumour resection was performed at the edge of the tunnel of a Signa SP 0.5-Tesla MRI. The surgeon aimed at a radical tumour resection that was followed by a peri-operative MRI scan. When a residual tumour was visualised and deemed resectable, an extended
\end{abstract}

This article is discussed in the editorial available at doi:10.1007/ s00701-011-1005-6.

J. Ramm-Pettersen $(\varangle) \cdot J$ J. Berg-Johnsen $\cdot$ E. Helseth

Department of Neurosurgery, Oslo University Hospital (OUS),

0027 Oslo, Norway

e-mail: jon.ramm-pettersen@oslo-universitetssykehus.no

J. Berg-Johnsen • E. Helseth

Faculty of Medicine, University of Oslo,

Oslo, Norway

P. K. Hol · S. Roy

The Intervention Center, OUS,

Oslo, Norway

J. Bollerslev $\cdot$ T. Schreiner

Section for Endocrinology, Clinic of Medicine, OUS,

Oslo, Norway resection was performed, followed by another MRI scan. This procedure was repeated until the imaging results were satisfactory. In all patients, we were able to obtain images to assess the extent of resection and to classify the resection as either total or subtotal.

Results After primary resection, eight out of 20 cases were classified as total resections. A second resection was performed in 11 of 12 cases classified as subtotal resections, and in four of these, total resection was achieved. A third resection was performed in three of the remaining seven cases with subtotal resections, but we did not achieve total resection in any of these cases. Therefore, the use of iMRI increased the number of patients with total resection from $8 / 20(40 \%)$ to $12 / 20(60 \%)$. The only observed complication was a transient spinal fluid leakage. Conclusion Intra-operative MRI during trans-sphenoidal microsurgery is useful in selected patients for a safe and more complete resection.

Keywords Pituitary · Intra-operative MRI . Trans-sphenoidal $\cdot$ Surgery

\section{Introduction}

Approximately $95 \%$ of pituitary adenomas that require surgery can be approached through the trans-sphenoidal approach, whereas other tumours need a transcranial approach. The trans-sphenoidal approach is a minimally invasive, microsurgical approach that provides good access to the sella and the clivus; however, it has the limitation of no direct visualisation of the suprasellar and parasellar regions. After surgery for hormone-producing tumours, the hormonal activity levels act as a marker of the amount of remnant tumour. The remission rate for hormone-producing 
macroadenomas ranges from $40 \%$ to $56 \%$ in large series [20], indicating that a large portion of patients harbour tumour remnants. In non-functioning tumours, the extent of removal of the tumour is more difficult to assess. Early post-operative magnetic resonance imaging (MRI) are difficult to interpret due to post-operative changes in the limited operative field [11]. Published series on nonfunctioning pituitary adenomas show that radical tumour removal is achieved in $28-70 \%$ of patients $[2,8,11,21]$, depending on tumour size and invasiveness. Therefore, there is clearly a need for better visualisation and tools to improve the removal of tumours during trans-sphenoidal surgery.

Various visualisation modalities have been implemented to increase the degree of resection in trans-sphenoidal surgery, but sagittal fluoroscopy has been the only standard imaging during surgery in most departments. This type of imaging shows the position of the speculum and the instruments in relation to the bony landmarks. The infusion of air from a cervical puncture gives additional information during the visualisation of the diaphragm of the sella and may facilitate the descendens of the suprasellar portion of the tumour [13]. The use of transcranial ultrasound-guided trans-sphenoidal surgery has been reported, but it has not yet gained widespread acceptance [17, 19]. The most promising new method to facilitate the resection of pituitary adenomas is the use of an endoscope [9]. One of the first applications of intra-operative MRI (iMRI) was its use during trans-sphenoidal surgery [18]. Nevertheless, there are few publications focusing solely on the results of MRIguided trans-sphenoidal surgery in pituitary adenomas [3, $4,6,7,12,14,16,22]$.

Although the trans-sphenoidal approach is a minimally invasive, microsurgical approach, several complications are associated with this procedure. For example, injury to the carotid arteries is the most feared complication, and postoperative cerebrospinal fluid (CSF) leakage is the most frequent. The mortality rate of these patients is thought to be approximately $1 \%$, severe morbidity is thought to occur in $3-4 \%$, and lesser morbidity is observed in $4-5 \%$ of the patients [20].

The aim of the present study was to evaluate whether the use of iMRI improves tumour resection in trans-sphenoidal surgery for pituitary adenomas.

\section{Methods and materials}

Twenty patients with macroadenomas were selected for surgery with iMRI on the assumption that peri-operative imaging would be helpful during the procedure. Table 1 shows the patient characteristics. Of the 20 patients included in this study, five had been operated on previously using the trans-sphenoidal approach. Sixteen of the tumours were non-functioning adenomas, three secreted growth hormone, and one was a pharmaco-resistant prolactinoma. The maximum tumour diameter varied between 11 and 41 mm (mean $27 \mathrm{~mm}$ ). The Knosp classification of parasellar extension was used [10]. Three tumours were classified as Knosp grade 0 , one grade 1, seven grade 2, five grade 3 and four grade 4.

After anaesthesia was introduced (propofol and remiphentanyl) and proper monitoring established, the operating table was docked to the Signa SP 0.5-Tesla MRI. Coronal and sagittal T1-weighted and T2-weighted images without gadolinium were obtained before the start of surgery. Surgery was performed with the patient in the supine position with the head slightly elevated at the edge of the MRI tunnel (Fig. 1). All instruments were MRI-compatible, and the nasal speculum was designed to produce minimal artefacts during imaging (Æsculap, Tuttlingen, Germany). An MRI-compatible microscope with a video camera was used, and the operative images were transferred to monitors located in the operating theatre and in the MRI control room (Møller-Wedel, Hamburg, Germany). The endonasal trans-septal approach was used for the primary surgery, and the direct endonasal approach was used for the re-operations. The surgery was performed in a similar manner as that performed in the ordinary operating theatre. When the surgeon decided that the surgery was complete, an MRI scan was performed with the speculum in place, and the resection cavity was filled with saline. Careful haemostasis was performed prior to imaging, and T1-weighted and T2-weighted images in the coronal and sagittal planes without contrast were obtained. Figure 2 shows examples of the pre-operative, peri-operative and post-operative images.

The iMRI scanning time was $3 \mathrm{~min}$ and $30 \mathrm{~s}$ per series performed. The pre-operative and intra-operative scans consisting of T1-coronal, T1-sagittal, T2-coronal and T2sagittal, lasted approximately $14 \mathrm{~min}$. After the completion of the surgery, we added T1-coronal and T1-sagittal series with contrast, so that the post-operative scan-time was 21 min. In addition to the time consumption of the scans, we needed approximately $3 \mathrm{~min}$ for transportation in and out of the scanner. Thus, in a typical case with one re-exploration, the use of iMRI added approximately $60 \mathrm{~min}$ of operating time.

If a surgically resectable tumour was observed in the peri-operative images, an extended resection was performed, followed by a new imaging sequence. If no further tumour was recognised, or if the remnant was deemed not to be surgically accessible, a final MRI scan with coronal and sagittal T1 images with gadolinium was performed as a post-operative control. When these images were evaluated as having no remnant tumour, the speculum was removed, the floor of the sella was closed with a dura substitute and 
Acta Neurochir (2011) 153:1367-1373

1369

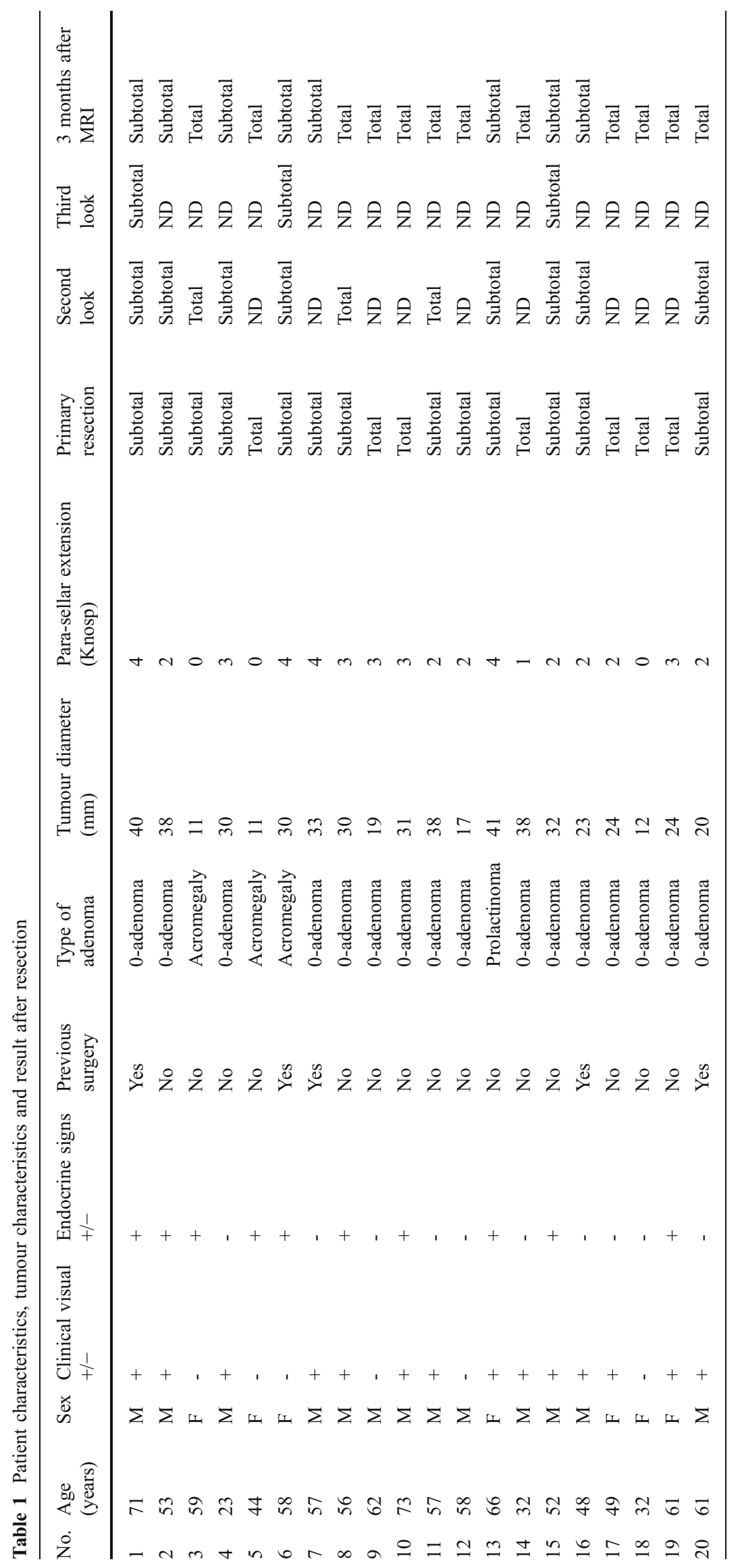

Springer 


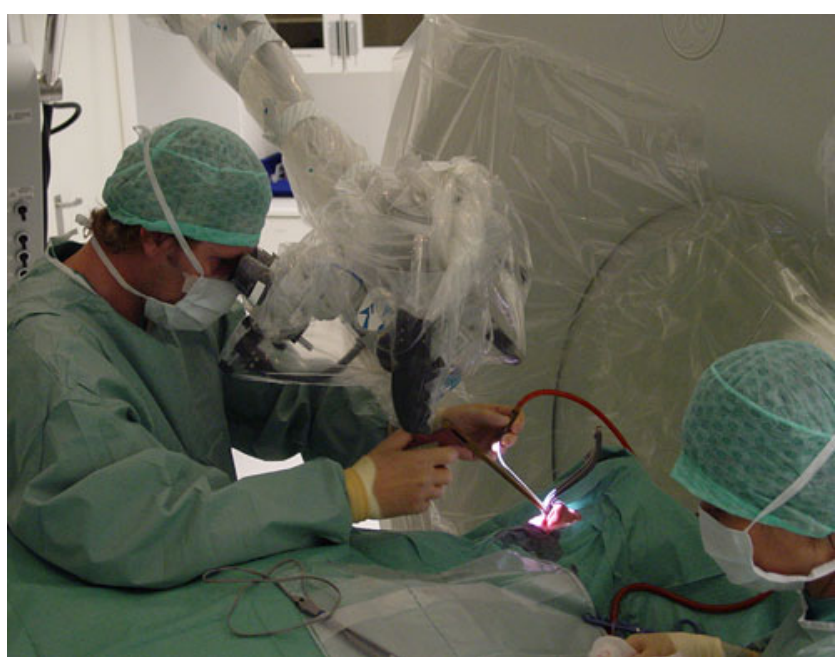

Fig. 1 The surgical set-up with the patient in the supine position. The patient's head was slightly elevated and rotated and was positioned just outside the MRI scanner. All the instruments were MRIcompatible, including the microscope. During imaging, the speculum was left in place, the resection cavity was filled with saline, and the patient was moved approximately $50 \mathrm{~cm}$ into the scanning position

bone from the midline or LactoSorb plates, and the sphenoid sinus was packed with gel foam.

All patients were seen for a 3-month follow-up at the Endocrinology Department. The evaluation included an assessment of post-operative pituitary function, an ophthalmologic examination, and a MRI scan. The pituitary function was assessed by tests for basal hormone values, 24-h urinary free cortisol and insulin tolerance. All MRI scans were evaluated by the two participating neuroradiologists. The coronal and sagittal $\mathrm{T} 1, \mathrm{~T} 2$ and $\mathrm{T} 1$ with gadolinium scans that were performed after the completion of surgery were evaluated and graded as either showing tumour remnant or showing radical removal.

\section{Results}

In all patients, images were obtained that could be used to assess the extent of resection. No re-exploration was performed in nine of the 20 patients. One re-exploration was performed in eight patients, and two re-explorations were performed in three patients (Table 1). In all 11 patients where re-exploration was performed, the procedure resulted in the removal of additional tumour.

\section{Extent of tumour resection}

Using the MRI results from the 3-month follow-up as the outcome measurement regarding the extent of resection, 12 out of the 20 patients were free of tumour remnants $(60 \%)$. In

Fig. 2 MRI pictures from the surgery of patient 4 , who had bilateral visual field deficits and was normopit pre-operative. At 3 months follow-up, the visual fields were normalised, and the patient was still normopit. a Pre-operative T1 without contrast in the Signa SP 0.5Tesla scanner. b Intra-operative T2 after primary resection. c Intra-operative $\mathrm{T} 1$ with contrast enhancement after extended resection showing a suspect remnant in the right side of the sella. d Three months post-operative follow-up showing subtotal resection with a remnant in the right side of the sella
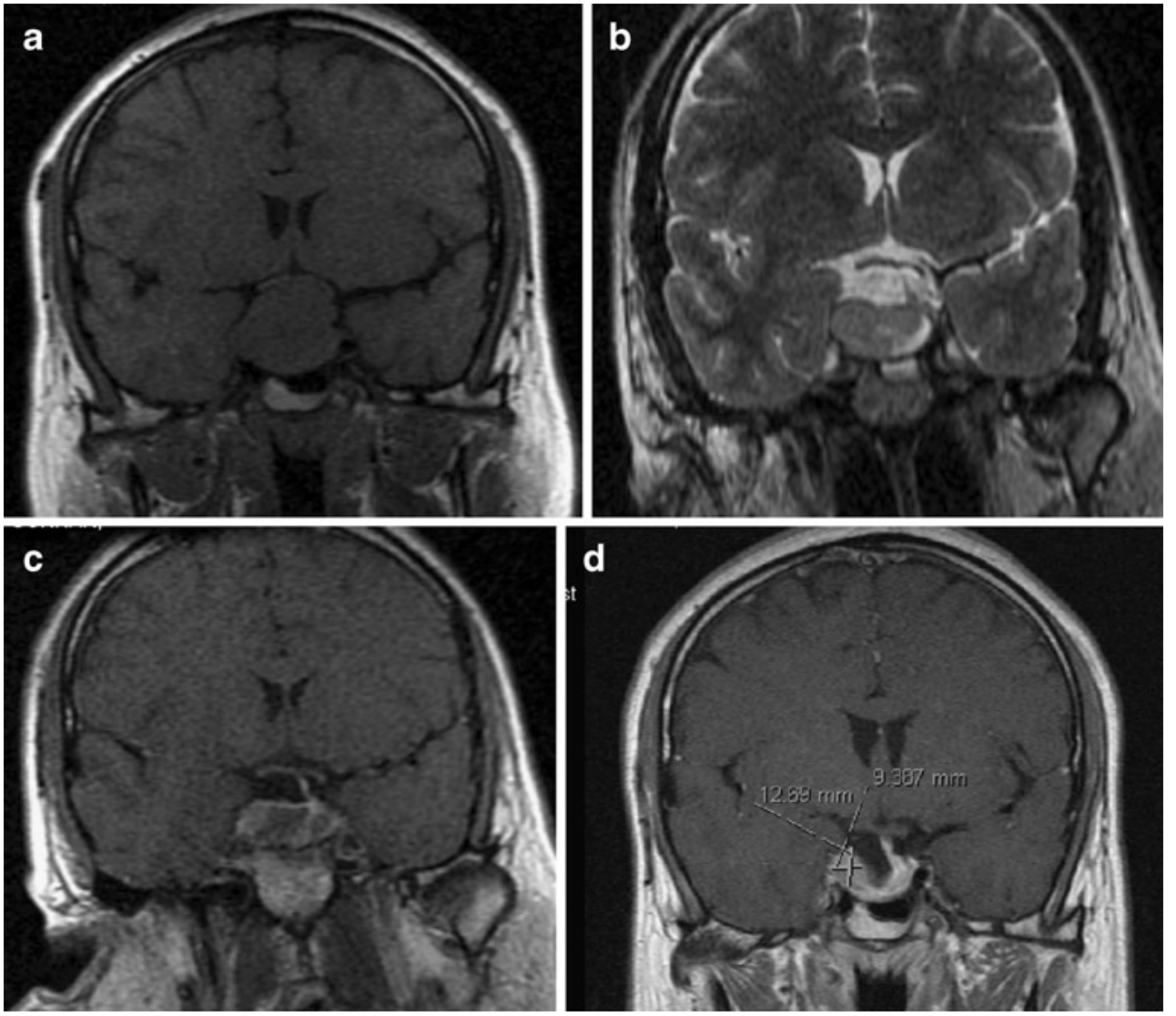
18 of the 20 patients, the extent of resection was similar when evaluated immediately post-operatively and at 3 months postoperatively. In two patients, a suspected remnant was observed in the post-operative scans, whereas at the 3-month follow-up, no tumour remnant was visible. Radical surgery was achieved in 12 patients: without re-exploration in eight, and with one re-exploration in the remaining four. We did not achieve radical surgery in any of the patients who underwent two re-explorations (Table 1). The mean tumour diameter in the group with total resection was $22 \mathrm{~mm}$, compared with 33 $\mathrm{mm}$ in patients with tumour remnants. The mean Knosp grade in the patients with radical surgery was 1.75 , and the mean grade was 3.13 in the group with tumour remnants. In all patients without parasellar extension (grade 0 or 1), we were able to extirpate the tumour. We achieved extirpation in $57 \%$ of the grade 2 patients, in $80 \%$ of the grade 3 patients, and in none of the grade 4 patients (Table 2).

In eight patients (40\%), the tumour was removed completely without re-exploration. With radical surgery at 3 months follow-up as the goal of the treatment, we found that the risk of a false-positive result was $10 \%$ (two out of 20 ) and that there was no risk of a false-negative result.

\section{Endocrinology}

Pre-operatively, ten patients had normal pituitary hormonal activity, seven patients had deficits in one or more of the pituitary hormonal axes (including the patient with prolactinoma), and three patients had growth-hormone hypersecretion (pituitary acromegaly). Eight of the ten patients with normal pituitary function pre-operatively retained normal pituitary function after surgery. One patient developed a growth-hormone deficiency, and one patient developed growth hormone and ACTH deficiencies. Of the seven patients with pre-operative hormonal dysfunction, three had normalised pituitary hormonal secretion at 3 months follow-up, whereas four had persistent pituitary hormonal dysfunction. Two of the three patients with growthhormone hypersecretion caused by a macroadenoma were cured after trans-sphenoidal surgery, and both of these patients had total resection on MRI evaluation. One patient

Table 2 Number of patients with no visible tumour on MRI at 3 months follow-up

\begin{tabular}{llll}
\hline $\begin{array}{l}\text { Knosp } \\
\text { grade }\end{array}$ & $\begin{array}{l}\text { No. } \\
\text { initial }\end{array}$ & $\begin{array}{l}\text { No. with total } \\
\text { resection }\end{array}$ & $\begin{array}{l}\text { Percent total } \\
\text { resection }\end{array}$ \\
\hline 0 & 3 & 3 & $100 \%$ \\
1 & 1 & 1 & $100 \%$ \\
2 & 7 & 4 & $57 \%$ \\
3 & 5 & 4 & $80 \%$ \\
4 & 4 & 0 & $0 \%$ \\
\hline
\end{tabular}

with acromegaly and subtotal resection exhibited growthhormone hypersecretion after surgery.

Ophthalmology

Fourteen of the patients in this study had visual field deficits prior to surgery. The visual field deficits improved in 12 of these patients after surgery $(86 \%)$, and two of the patients had unchanged visual fields at 3 months follow-up. Five patients had reduced visual acuity before surgery, and all of the patients had improved visual acuity at 3 months follow-up.

\section{Complications}

No haematomas and no infections were encountered in these patients. One patient had a post-operative CSF leak that resolved after 4 days of treatment with lumbar drainage.

\section{Discussion}

Since iMRI was introduced more than 15 years ago, many scanners and surgical techniques have been developed [1]. Most of the scanner configurations that have been described require transportation of either the patient or the scanner, which is time-consuming, labour-intensive, and makes it difficult to maintain sterility and anaesthesia. The Signa SP/ i 0.5-Tesla scanner that was used in this study was installed at our institution in 1996 and has been used mainly for biopsies and glioma surgeries. The 'double-doughnut' configuration of this scanner is designed to make it possible to operate inside the imaging field. The disadvantages of this scanner are its relatively low field strength of 0.5 Tesla and the requirement for non-magnetic instruments and microscopes. We have developed a system in which we operate just outside the MRI tunnel and slide the patient approximately $50 \mathrm{~cm}$ into the scanning position. We wanted to investigate the feasibility, safety and usefulness of this system during trans-sphenoidal surgery.

High-quality images were obtained in all patients, at the expected quality of the 0.5 -Tesla field strength for the T1 and T2 sequences. Fahlbusch et al. [6] reported that the evaluation of the intra-operative images was obscured by an artefact in 13 of 44 patients (30\%) due to metal debris from drilling or from blood in the cavity. We used a scissile to open the sella instead of a high-speed drill during surgery in the open MRI. The intra-operative imaging was performed without any closing of the sella and without any marker, like wax or cotton pledges, in the resection cavity. After thorough haemostasis, we filled the resection cavity and the speculum with isotonic saline. Remnant tumours and capsule membranes were visualised on $\mathrm{T} 2$ images as areas 
of low signal intensity between the high signal intensity in the basal cisterns and the fluid-filled resection cavity.

We achieved primary radical surgery in $40 \%$ of the patients (eight out of 20), which correlates well with other published data reporting primary radical surgery in $27 \%$ [6] and $34 \%$ of patients [3]. Nevertheless, these figures cannot be compared directly because of the heterogeneity of the groups. In addition, we have shown that complete resection is more easily achieved in smaller tumours that have little to no parasellar extension.

The use of iMRI increased the percentage of patients that received radical surgery from $40 \%$ to $60 \%$. The patients that were included in this study were selected on the assumption that intra-operative imaging would aid in complete resection during surgery, especially for the instances in which the tumours had a parasellar or suprasellar extension, which predicted that parts of the tumour would not be directly visualised by the microscope in the operating field. If we had selected tumours that were located more in the centre of the sella, we would probably have achieved a higher percentage of patients with total resection; however, intra-operative imaging would have likely been less useful in these circumstances. When comparing the results from studies of trans-sphenoidal surgery, it is necessary to note the size of the tumours and the degree of parasellar extension.

Eight of ten patients exhibited normal pituitary hormonal activity after surgery (80\%).

In the small subgroup of patients with growth-hormoneproducing tumours (three patients), there was a $100 \%$ correlation between the intra-operative imaging results and the post-operative hormonal status. Two patients had no residual tumour, and they both exhibited a biochemical cure. The third patient, who had been operated on earlier and had a parasellar extension of the tumour (Knosp grade 4), had a tumour remnant on intra-operative imaging and showed growth-hormone hypersecretion at 3 months follow-up. Other publications have failed to show a similar correlation in acromegalic patients [7].

The design of this study was based on the presumption that the surgeon has performed as complete a resection of the tumour as possible before the iMRI scans are performed. Nevertheless, it is possible that the surgeon was more conservative during resection because an iMRI was available. The assumption that an iMRI leads to a more complete resection can thus be a self-fulfilling prophecy. The only way to avoid this bias is to design a study in which the tumours are randomised to either standard microsurgical trans-sphenoidal surgery or to trans-sphenoidal surgery with iMRI and to ensure that the groups are comparable in regard to tumour size and the degree of parasellar extension.

During treatment of hormone-producing tumours, it is important to strive for radicality in resection and to thereby cure the patient of a devastating disease. When treating non-producing tumours, however, a complete resection may not be as important. Striving for radicality in the resection increases the risk of complications during surgery, including post-operative CSF leakage, new endocrinologic deficits and haemorrhage. We reviewed the outcomes of pituitary surgeries in patients older than 70 years in our department and found that they had excellent outcomes on both mortality and performance scales, even though many of these patients had remnant tumours [15].

Traditionally, the usefulness of early MRI validation of trans-sphenoidal surgery has been questioned due to difficulty in interpreting the images. Precluding factors in this procedure can include the material used to close the sella, the haemostatic agents left in the cavity, and the difficulties in discerning blood from the tumour remnants and discerning the re-expanded pituitary gland from the tumour tissue [5]. Our experience with iMRI has taught us to use T1 with contrast enhancement and T2 as a post-operative control. At our institution, an early MRI control is performed within $48 \mathrm{~h}$ after all transsphenoidal procedures that are not performed in the iMRI. Learning from this objective evaluation of the extent of tumour resection immediately after surgery is extremely important, especially for less experienced surgeons.

\section{Conclusion}

Intra-operative MRI is a useful tool during trans-sphenoidal surgery for pituitary adenomas. Using this procedure, good results were achieved in regard to the extent of tumour resection and endocrinologic and visual outcomes, with a low complication rate. Learning from an objective evaluation of the extent of resection immediately after surgery is important, especially for less experienced surgeons, and may improve the results after trans-sphenoidal surgery.

\section{Conflicts of interest None.}

Open Access This article is distributed under the terms of the Creative Commons Attribution Noncommercial License which permits any noncommercial use, distribution, and reproduction in any medium, provided the original author(s) and source are credited.

\section{References}

1. Albayrak B, Samdani AF, Black PM (2004) Intra-operative magnetic resonance imaging in neurosurgery. Acta Neurochir 146:543-557

2. Alameda C, Lucas T, Pineda E, Brito M, Uria JG, Magalon R, Estrada J, Barcelo B (2005) Experience in management of 51 non- 
functioning pituitary adenomas: indications for post-operative radiotherapy. J Endocrinol Invest 28(1):18-22

3. Bohinski RJ, Warnick RE, Gaskill-Shiplev MF, Zuccarello M, van Loveren HR, Kormos DW, Tew JM Jr (2001) Intraoperative magnetic resonance imaging to determine the extent of resection of pituitary macroadenomas during transsphenoidal microsurgery. Neurosurgery 49(5):1133-1143

4. Darakchiev BJ, Tew JM Jr, Bohinski RJ, Warnick RE (2005) Adaptation of a standard low-field $(0.3 \mathrm{~T})$ system to the operating room: focus on pituitary adenomas. Neurosurg Clin N Am 16 (1):155-164

5. Dina TS, Feaster SH, Laws ER Jr, Davis DO (1993) MR of the pituitary gland postsurgery: serial MR studies following transsphenoidal resection. AJNR Am J Neuroradiol 14(3):763-769

6. Fahlbusch R, Ganslandt O, Buchfelder M, Schott W, Nimsky C (2001) Intraoperative magnetic resonance imaging during transsphenoidal surgery. J Neurosurg 95:381-390

7. Fahlbusch R, Keller B, Ganslandt O, Kreutzer J, Nimsky C (2005) Transsphenoidal surgery in acromegaly investigated by intraoperative high-field magnetic resonance imaging. Eur J Endocrinol 153(2):239-248

8. Greenman Y, Ouaknine G, Veshchev I (2003) Postoperative surveillance of clinically non-functioning pituitary macroadenomas: markers of tumour quiescence and regrowth. Clin Endocrinol 58(6):763-769

9. Kabil MS, Eby JB, Shahinian HK (2005) Fully endocopic endonasal vs. transseptal transsphenoidal pituitary surgery. Minim Invasive Neurosurg 48(6):348-354

10. Knosp E, Steiner E, Kitz K, Matula C (1993) Pituitary adenomas with invasion of the cavernous sinus space: a magnetic resonance imaging classification compared with surgical findings. Neurosurgery 33:610-618

11. Kremer P, Forsting M, Ranaei G, Wurster C, Hamer J, Sartor K, Kunze S (2002) Magnetic resonance imaging after Transsphenoidal Surgery of clinically non-functional pituitary macroadenomas and its impact on detecting residual adenoma. Acta Neurochir 144:433-443

12. Martin CH, Schwartz R, Jolesz F, Black PM (1999) Transsphenoidal resection of pituitary adenomas in an intraoperative MRI unit. Pituitary 2(2):155-162

13. Nesbakken R, Reinlie S, Eldevik OP (1984) Intraoperative gas cisternography and gas dissection in the operative treatment of pituitary tumors. A methodological description. Eur Surg Res 16 (Suppl 2):73-79

14. Pergolizzi RS Jr, Nabavi A, Schwartz RB, Hsu L, Wong TZ, Martin C, Black PM, Jolesz FA (2001) Intra-operative MR guidance during trans-sphenoidal pituitary resection: preliminary results. J Magn Reson Imaging 13(1):136-141

15. Rogne SG, Konglund A, Meling TR, Scheie D, Johannesen TB, Rønning P, Helseth E (2009) Intracranial tumor surgery in patients $>70$ years of age: is clinical practice worthwhile or futile? Acta Neurol Scand 120(5):288-294

16. Schwartz TH, Stieg PE, Anand VK (2006) Endoscopic transsphenoidal pituitary surgery with intraoperative magnetic resonance imaging. Neurosurgery 58( 1 Suppl):ONS44-ONS51

17. Solheim O, Selbekk T, Løvstakken L, Tangen GA, Solberg OV, Johansen TF, Cappelen J, Unsgård G (2010) Intrasellar ultrasound in transsphenoidal surgery: a novel technique. Neurosurgery 66 (1):173-185

18. Steinmeier R, Fahlbusch R, Gansland O, Nimsky C, Buchfelder M, Kaus M, Heigl T, Lenz G, Kuth R, Huk W (1998) Intraoperative magnetic resonance imaging with the magnetom open scanner: concepts, neurosurgical indications, and procedures: a preliminary report. Neurosurgery 43(4):739-747

19. Suzuki R, Asai J, Nagashima G, Itokawa H, Chang CW, Noda M, Fujimoto M, Fujimoto T (2004) Transcranial echo-guided transsphenoidal surgical approach for the removal of large macroadenomas. J Neurosurg 100(1):68-72

20. Thapar K, Laws ER. (2004) Pituitary tumors: functioning and nonfunctioning. In: Youmans Neurological surgery, 5th edn. Saunders (Elsevier), Philadelphia, pp 1169-1206

21. Wischer-Rother M, Hoven S, Kristoff RA, Bliesener N, StoffelWagner B (2004) Non-functioning pituitary adenomas: endocrinological and clinical outcome after transsphenoidal and transcranial surgery. Exp Clin Endocrinol Diabetes 112(6):323327

22. Wu JS, Shou XF, Yao CJ, Wang YF, Zhuang DX, Mao Y, Li SQ, Zhou LF (2009) Transsphenoidal pituitary macroadenomas resection guided by PoleStar N20 low-field intraoperative magnetic resonance imaging: comparison with early postoperative high-field magnetic resonance imaging. Neurosurgery 65 (1):63-70 